\title{
A calibration of the 85 Peg binary system
}

\author{
J. Fernandes ${ }^{1}$, P. Morel $^{2}$, and Y. Lebreton ${ }^{3}$ \\ ${ }^{1}$ Observatório Astronómico da Universidade de Coimbra, Santa Clara, 3040 Coimbra, Portugal and Departamento \\ de Matemática da Faculdade de Ciências e Tecnologia da Universidade de Coimbra, Portugal \\ 2 Département Cassini, UMR CNRS 6529, Observatoire de la Côte d'Azur, BP 4229, 06304 Nice Cedex 4, France \\ ${ }^{3}$ GEPI, Observatoire de Paris Meudon, 92195 Meudon, France
}

Received 28 September 2001 / Accepted 11 June 2002

\begin{abstract}
We have calibrated the initial abundances, age and mixing-length parameters of the visual binary system 85 Pegasi. We obtain an age $t_{85} \mathrm{Peg}=9345 \pm 500 \mathrm{Myr}$, masses $m_{\mathrm{A}}=0.88 \pm 0.01 M_{\odot}$ and $m_{\mathrm{B}}=0.55 \pm 0.02 M_{\odot}$, an initial helium mass fraction $Y_{\mathrm{i}}=0.25 \pm 0.01$, an initial metallicity $\left[\frac{\mathrm{Fe}}{\mathrm{H}}\right]_{\mathrm{i}}=-0.185 \pm 0.054$ and mixing-length parameters $\Lambda_{\mathrm{A}}=1.80 \pm 0.05$ and $\Lambda_{\mathrm{B}}=2.14 \pm 0.10$. We find that, as already proposed, $85 \mathrm{Peg} \mathrm{B}$ is itself a binary. The mass of the unseen companion is $m_{\mathrm{b}} \approx 0.11 M_{\odot}$
\end{abstract}

Key words. stars: binaries: visual - stars: evolution - stars: fundamental parameters - stars: individual: 85 Peg

\section{Introduction}

The calibration of a binary system is based on the adjustment of stellar modeling parameters $\left(t_{\star}, Y_{\mathrm{i}},\left[\frac{\mathrm{Fe}}{\mathrm{H}}\right]_{\mathrm{i}}, \Lambda_{\mathrm{A}}, \Lambda_{\mathrm{B}}\right)$ to the observational data at the age $t_{\star}$ of the system, with the reasonable hypothesis of a common origin for both components (same initial chemical composition and age). $Y_{\mathrm{i}}$ and $\left[\frac{\mathrm{Fe}}{\mathrm{H}}\right]_{\mathrm{i}}$ are, respectively, the initial helium mass fraction and metallicity, $\Lambda_{\mathrm{A}}$ and $\Lambda_{\mathrm{B}}$ are respectively the mixing-length parameters of the primary A and secondary B. For each component one also infers a mass value consistent with theoretical stellar modeling.

Taking into account the most recent theoretical and observational astrometric, spectroscopic and photometric results we undertake the calibration of 85 Pegasi (HD 224930, HIP 171, BD+2647344, HR 9088, $\beta 733$ ADS 17175, IDS 23569+2633; $\alpha=00^{\mathrm{h}} 02^{\mathrm{m}} 10^{\mathrm{s}}, \delta=+27^{\circ} 04^{\prime} 56^{\prime \prime}(2000)$ ). It is a well-known visual and single-lined spectroscopic binary system. The components, 85 Peg A and B, are main sequence low mass stars of spectral types G5 and K7 respectively (ten Brummelaar et al. 2000). The shortness of the period ( $\approx 27$ years) and the proximity to the Sun $(\sim 12 \mathrm{pc})$ give it an interesting place among the binary stars. The visual orbit is very well known (Hall 1948). Nevertheless, some studies of the orbit have been recently published. The latest (Söderhjelm 1999) has yielded improvements to the Hipparcos trigonometric parallax and orbit, combining data from the satellite with ground-based observations. $85 \mathrm{Peg}$ is one of the rare cases for which the mass ratio can be obtained by both astrometric and spectroscopic observations. For many years investigators have claimed that $85 \mathrm{Peg} \mathrm{B}$, the fainter component, is more

Send offprint requests to: J. Fernandes, e-mail: jmfernan@mat.uc.pt massive than 85 Peg A the brighter one (e.g. Slocum 1915; Hall 1948; Underhill 1963). This was found again by Martin \& Mignard (1998) on the basis of Hipparcos observations. This abnormal situation could be explained by the fact that 85 Peg B is an undetected binary. $85 \mathrm{Peg} \mathrm{A}$ is known as a metal-poor star and found to be much older than the Sun (Perrin et al. 1977). Because of its small mass, 85 Peg A sits close to the zams which gives a constraint on its initial helium abundance, a value traditionally associated with primordial helium (Perrin et al. 1977; Catchpole et al. 1967; Smak 1960).

The photometry and the atmospheric parameters of 85 Peg A have been determined many times. The first spectroscopic determination of the metallicity is from Wallerstein \& Helfer (1959), while the most recent metallicity and effective temperature determinations are due to van't Veer (2000) and Fulbright (2000). While 85 Peg A is a well known star, the secondary component is less well known. The greatest limitations to studying 85 Peg B in detail were the lack of individual photometric and spectroscopic measurements due to its proximity to 85 Peg A $(\rho \approx 0$ ' 75$)$. Recently, ten Brummelaar et al. (2000), performed differential photometry in the Johnson VRI system for both stars using adaptative optics, which allows us to determine the effective temperatures and bolometric magnitudes of both components.

In their pioneering work Fernandes et al. (1998) tried to calibrate the helium abundance and age of both 85 Peg A and B by means of stellar models. No solution was possible when fitting both components for the same helium and age values. The models appeared to be hotter than the observations. The discrepancy appears to be removed if the microscopic diffusion of helium and heavy elements, the enrichment of $\alpha$-elements and the non-LTE effects in the iron abundances determinations 
Table 1. Relevant orbital elements of the $85 \mathrm{Peg}$. As usual $P$ is the orbital period in years, $a$ the semi-major axis, $i$ the inclination and $e$ the eccentricity. The error bars are probable errors. Söderhjelm does not give the errors on the orbital elements but he indicates that the number of given decimals reflect the mean errors.

\begin{tabular}{lllll}
\hline \hline Author & $P$ & $a$ & $i$ & $e$ \\
\hline Hall (1948) & $26.27 \pm 0.19$ & $00^{\prime} 83 \pm 00^{\prime} 02$ & $-50^{\circ} 0 \pm 2.0$ & $0.38 \pm 0.01$ \\
Söderhjelm (1999) & 26.28 & 0.83 & -49. & 0.38 \\
\hline
\end{tabular}

are taken into account in models of low mass stars (Lebreton et al. 1999). Nevertheless, Lebreton et al. did not perform a full calibration of $85 \mathrm{Peg} \mathrm{A}$ and did not examine the secondary component.

Our purpose here is (1) to provide a complete modeling of the system based on more appropriate stellar models (i.e. including microscopic diffusion) and (2) to propose a calibration of the unknown parameters (age, helium, individual masses) that fulfills the recent observational constraints.

The paper is divided as follows. In Sect. 2, we collect and discuss the observational constraints. Section 3 is devoted to the description of physics and modeling. We summarize and conclude in Sect. 4.

\section{Observations of the visual binary $85 \mathrm{Peg}$}

\subsection{Astrometric data}

For $85 \mathrm{Peg}$ we are in the fortunate position of having two precise, self consistent and independent determinations of the trigonometrical parallax and improved orbital elements; from these data individual masses $m_{\mathrm{A}}$ and $m_{\mathrm{B}}$ can be derived independently. Table 1 shows the excellent agreement of the relevant elements of the astrometric orbits of Hall (1948) and Söderhjelm (1999). The first determination of the parallax is the standard photographic parallax (Wyller 1956; Heintz 1993) based on improved orbit and several decades of photographic long focus observations: the absolute photographic parallax amounts to $\varpi_{\mathrm{abs}}=0 .{ }^{\prime} 0798 \pm 00^{\prime} 0033$ (Heintz 1993). The second determination is the recently improved adjustment of parallax and orbital elements by Söderhjelm (1999) based on the Hipparcos $3.25 \mathrm{yr}$ data and old ground-based observations yielding $\varpi_{\text {abs }}=00^{\prime} 0825 \pm 0$.'0008. With the orbit of Hall (1948) and the parallax of Heintz (1993) we derive the sum of masses $\mathcal{S}_{85 \text { Peg }}=1.63 \pm 0.33 M_{\odot}$. Using the data from Söderhjelm (1999) we obtain $\mathcal{S}_{85 \text { Peg }}=1.49 \pm 0.09 M_{\odot}$. For the sum of masses we adopt the weighted average value:

$\mathcal{S}_{85 \mathrm{Peg}}=1.50 \pm 0.09 M_{\odot}$.

An estimate of the mass fraction $B \equiv m_{\mathrm{B}} /\left(m_{\mathrm{A}}+m_{\mathrm{B}}\right)$ is provided by long-focus astrometry (Heintz 1993):

$B_{\mathrm{p}}=0.44 \pm 0.01$ (p.e.).

Another estimate of $B$ is provided by the spectroscopic orbit (e.g. Underhill 1963). Combined spectroscopic observations covering about four decades and astrometric measurements give the velocity semi-amplitude of the primary $K_{\mathrm{A}}=4.10 \pm 0.15 \mathrm{~km} \mathrm{~s}^{-1}$ (Duquennoy \& Mayor 1991). With the adopted orbital elements and parallax, we derive the "spectroscopic" mass fraction:

$B_{\mathrm{s}}=0.43 \pm 0.06$.

Note the remarkable agreement between spectroscopic and photographic determinations of the mass fraction. A weighted average of the photographic and spectroscopic values leads to:

$B_{85 \text { Peg }}=0.44 \pm 0.02$.

With the sum of masses and mass fraction derived above, the masses of the components are respectively:

$m_{\mathrm{A}}=0.84 \pm 0.08 M_{\odot}, \quad m_{\mathrm{B}}=0.66 \pm 0.07 M_{\odot}$,

showing 85 Peg A definitively to be the more massive star.

\subsection{Spectroscopic and photometric data}

Stellar parameters were determined from spectroscopic and photometric analysis. Table 2 lists recently published data for the photometry, effective temperature and metallicity of $85 \mathrm{Peg}$.

For 85 Peg A we chose to retain the recent determinations $\left[\frac{\mathrm{Fe}}{\mathrm{H}}\right]_{\mathrm{A}}=-0.69 \mathrm{dex}$ and $T_{\text {eff A }}=5550 \mathrm{~K}$ of van't Veer $(2000)$. This $T_{\text {eff }}$ determination relies on the careful fit of Balmer line profiles with Kurucz's (1991) ATLAs model atmospheres with the constraint that the model reproduces both the $\mathrm{H}_{\alpha}$ and $\mathrm{H}_{\beta}$ profiles. Furthermore we point out that, according to Thévenin \& Idiart (1999), the metallicity of metal-poor stars, computed using model atmospheres in the LTE approximation is underestimated by about 0.12 dex at the metallicity of $85 \mathrm{Peg}$. This is particularly important for the stellar position on the HR diagram (Lebreton et al. 1999). We take into account this correction and adopt the value $\left[\frac{\mathrm{Fe}}{\mathrm{H}}\right]_{\mathrm{A}}=-0.57 \pm 0.11$ (Thévenin 2001). The $\alpha$-elements ( $\mathrm{Mg}, \mathrm{Ca}, \mathrm{Si}, \mathrm{Na}, \mathrm{Al}, \mathrm{Ti})$ have been found to be enriched with respect to the Sun in 85 Peg A (Fuhrmann 1998; van't Veer 2000; Fulbright 2000). In our calculations we take account of an $\alpha$-elements enrichment, $[\alpha / \mathrm{Fe}]=+0.40 \mathrm{dex}$, through appropriate opacities and input mixture. We computed the effective temperature of 85 Peg B using the calibrations $T_{\text {eff }}=T_{\text {eff }}\left\{\right.$ color, $\left.\left[\frac{\mathrm{Fe}}{\mathrm{H}}\right]\right\}$ from Lejeune et al. (1998) and Bessell et al. (1998). We use both the color index $(R-I)$ and $(V-I)$ from ten Brummelaar et al. (2000). These indices are not very sensitive to the metallicity (Alonso et al. 1996). We obtain $T_{\text {eff B }}=4100 \mathrm{~K}$ using $(R-I)$ and $T_{\text {eff B }}=4300 \mathrm{~K}$ using $(V-I)$. We choose $T_{\text {eff B }}=4200 \mathrm{~K}$ as a representative value of the effective temperature for the secondary.

The luminosities are computed using the photometry of ten Brummelaar et al. (2000), the corrected Hipparcos parallax from Söderhjelm (1999) and the bolometric correction either from Bessell et al. (1998) or from Flower (1996). Using 
Table 2. Photometric and spectroscopic data for 85 Peg.

\begin{tabular}{lll}
\hline \hline & 85 Peg A & 85 Peg B \\
\hline$V^{1}$ & $5.81 \pm 0.03$ & $8.89 \pm 0.29$ \\
$R^{1}$ & $5.25 \pm 0.03$ & $7.95 \pm 0.27$ \\
$I^{1}$ & $4.85 \pm 0.03$ & $7.20 \pm 0.26$ \\
& & \\
$T_{\text {eff }}{ }^{2}$ & $5524 \pm 50 \mathrm{~K}$ \\
$T_{\text {eff }}{ }^{3}$ & $5550 \pm 50 \mathrm{~K}$ \\
$T_{\text {eff }}{ }^{4}$ & $5275 \pm 100 \mathrm{~K}$ \\
& \\
{$\left[\frac{\mathrm{Fe}}{\mathrm{H}}\right]_{\mathrm{A}}{ }^{2}$} & $-0.86 \pm 0.06$ \\
{$\left[\frac{\mathrm{Fe}}{\mathrm{H}}\right]_{\mathrm{A}}{ }^{3}$} & $-0.69 \pm 0.05$ \\
{$\left[\frac{\mathrm{Fe}}{\mathrm{H}}\right]_{\mathrm{A}}{ }^{4}$} & $-0.9 \pm 0.1$ \\
\hline${ }^{1}$ ten Brummelaar et al. $(2000)$. \\
${ }^{2}$ Axer et al. (1994). \\
${ }^{3}$ van't Veer (2000). \\
${ }^{4}$ Fulbright (2000).
\end{tabular}

Table 3. Adopted calibration constraints.

\begin{tabular}{ll}
\hline \hline$S_{85 \text { Peg }}$ & $1.50 \pm 0.09 M_{\odot}$ \\
$B_{85 \text { Peg }}$ & $0.44 \pm 0.02$ \\
$T_{\text {eff A }}$ & $5550 \pm 50 \mathrm{~K}$ \\
$T_{\text {eff B }}$ & $4200 \pm 200 \mathrm{~K}$ \\
$L_{\mathrm{A}} / L_{\odot}$ & $0.616 \pm 0.02$ \\
$L_{\mathrm{B}} / L_{\odot}$ & $0.071 \pm 0.02$ \\
{$\left[\frac{\mathrm{Fe}}{\mathrm{H}}\right]_{\mathrm{A}}$} & $-0.57 \pm 0.11$ \\
\hline
\end{tabular}

Bessell et al.'s (1998) results we obtain $L_{\mathrm{A}} / L_{\odot}=0.611 \pm 0.040$ and $L_{\mathrm{B}} / L_{\odot}=0.069 \pm 0.030$ and with Flower's (1996) results we get $L_{\mathrm{A}} / L_{\odot}=0.622 \pm 0.040$ and $L_{\mathrm{B}} / L_{\odot}=0.072 \pm 0.030$. The agreement is satisfactory and we take the average values.

Table 3 lists the sum of masses, mass fraction, effective temperatures, luminosities and metallicities we selected to constrain the models.

\section{Modeling}

Stellar models have been computed using the CESAM code (Morel 1997). The physics of the models and the calibration method employed here are described in Morel et al. (2000); element diffusion and pre-main sequence evolution are taken into account. We search a solution with the $\chi^{2}$-minimization described by Lastennet et al. (1999) and Morel et al. (2000). The confidence limits of each modeling parameter, the other being fixed, correspond to the maximum/minimum values it can reach, in order that the generated models fit the observable targets within their error bars. Table 4 lists the set of modeling parameters we derive for 85 Peg A \& B. Figure 1 shows the corresponding evolutionary tracks in the HR diagram.

\section{Discussion and conclusion}

According to the $\chi^{2}$ minimization we achieved the first calibration of the visual binary system $85 \mathrm{Peg}$, i.e. we found a set of calibration parameters $\left(t_{\star}, Y_{\mathrm{i}},\left[\frac{\mathrm{Fe}}{\mathrm{H}}\right]_{\mathrm{i}}, \Lambda_{\mathrm{A}}, \Lambda_{\mathrm{B}}\right)$ and theoretical masses $m_{\mathrm{A}}$ and $m_{\mathrm{B}}$ which give models that fit the observables
Table 4. Calibration parameters and global parameters of $85 \mathrm{Peg} \mathrm{A}$ and $\mathrm{B}$ models lying within the uncertainty boxes. The four first rows show the modeling parameters $t_{85 \mathrm{Peg}}$ is the age of the binary in Myr, $Y_{\mathrm{i}}$, $\left[\frac{\mathrm{Fe}}{\mathrm{H}}\right]_{\mathrm{i}}$ are the initial values of respectively the helium mass fraction and the metallicity, $\Lambda$ is the mixing-length parameter. The next six rows shows the theoretical values obtained for the observational constraints of Table 3 recalled in parenthesis, $\mathcal{S}$ is the sum of masses in solar unit, $B$ is the mass fraction, $T_{\text {eff }}$ in $\mathrm{K}$ and $L$ in solar unit are respectively the effective temperature and the luminosity, $\left[\frac{\mathrm{Fe}}{\mathrm{H}}\right]_{\mathrm{s}}$ is the surface metallicity. In next rows $m$ is the mass in solar unit, $R$ the radius in solar unit and $Y_{\mathrm{s}}$ the helium mass fraction at the surface.

\begin{tabular}{ccc}
\hline \hline & 85 Peg A & 85 Peg B \\
\hline$t_{85 P e g}$ & \multicolumn{2}{c}{$9345 \pm 500$} \\
$Y_{\mathrm{i}}$ & \multicolumn{2}{c}{$0.253 \pm 0.01$} \\
{$\left[\frac{\mathrm{Fe}}{\mathrm{H}}\right]_{\mathrm{i}}$} & \multicolumn{2}{c}{$-0.185 \pm 0.054$} \\
$\Lambda$ & $1.80 \pm 0.05$ & $2.14 \pm 0.10$ \\
$\mathcal{S}$ & \multicolumn{2}{c}{$1.43(1.50 \pm 0.09)$} \\
$B$ & $0.385(0.44 \pm 0.02)$ \\
$T_{\mathrm{eff}}$ & $5581(5550 \pm 80)$ & $4220(4200 \pm 200)$ \\
$L$ & $0.622(0.616 \pm 0.02)$ & $0.071(0.071 \pm 0.02)$ \\
{$\left[\frac{\mathrm{Fe}}{\mathrm{H}}\right]_{\mathrm{S}}$} & $-0.55(-0.57 \pm 0.11)$ & -0.49 \\
\multicolumn{3}{c}{} \\
$m$ & $0.88 \pm 0.01$ & $0.55 \pm 0.02$ \\
$R$ & 0.846 & 0.500 \\
$Y_{\mathrm{S}}$ & 0.216 & 0.237 \\
\hline
\end{tabular}

within their error bars. Table 4 and Fig. 1 show the calibration parameters and the evolutionary tracks in the HR diagram. The most amazing result concerns the masses in the system. Table 4 shows that the models predict the sum of masses with satisfactory agreement (error of $4.6 \%$ between models and observations). This is also true for the mass of $85 \mathrm{Peg} \mathrm{A}$ (error of $4.5 \%$ ). The fact that the observational mass of the main component is in agreement with the "astrophysical" one was already pointed out by Lebreton et al. (1999). On the other hand, the mass ratio is poorly reproduced. This has a direct influence on the prediction of the mass of 85 Peg B: $0.55 M_{\odot}$ from the models against $\sim 0.66 M_{\odot}$ from the observations. Our fit strongly suggests that $85 \mathrm{Peg} \mathrm{B}$ is itself a binary B-b, with a $m_{\mathrm{b}} \approx 0.11 M_{\odot}$ third component. The theoretical mass we derive for the fainter component significantly differs from the astrometric result by more than $1 \sigma$. Such disagreement between the mass determinations from astrometric and from stellar modeling reveals either erroneous data (observational or theoretical) or a too large astrometrical mass determination due to an unseen component. On the other hand, one reasonably expects a mass difference between the two main sequence stars 85Peg A \& B fulfilling the mass luminosity relationship (MLR). Using the MLR in the $V$ filter of Henry \& Mc Carthy (1993, formula 3a) one derives a mass difference of $\approx 0.35 \pm 0.08 M_{\odot}$ between the components, while the astrometric data predict $\approx 0.18 \pm 0.10 M_{\odot}$. This difference is consistent with the possibility of an unseen companion around the secondary. We emphasize that a star of $0.11 M_{\odot}$ is 35 times fainter than a $0.55 M_{\odot}$ star (Baraffe et al. 1998) and does not contribute to the luminosity of $85 \mathrm{Peg} \mathrm{B}+\mathrm{b}$, while it affects the astrometric mass determination. Figure 1 shows the evolutionary tracks for the models of $0.88 M_{\odot}$ and $0.55 M_{\odot}$ corresponding to our best solution. We also plot the evolutionary 

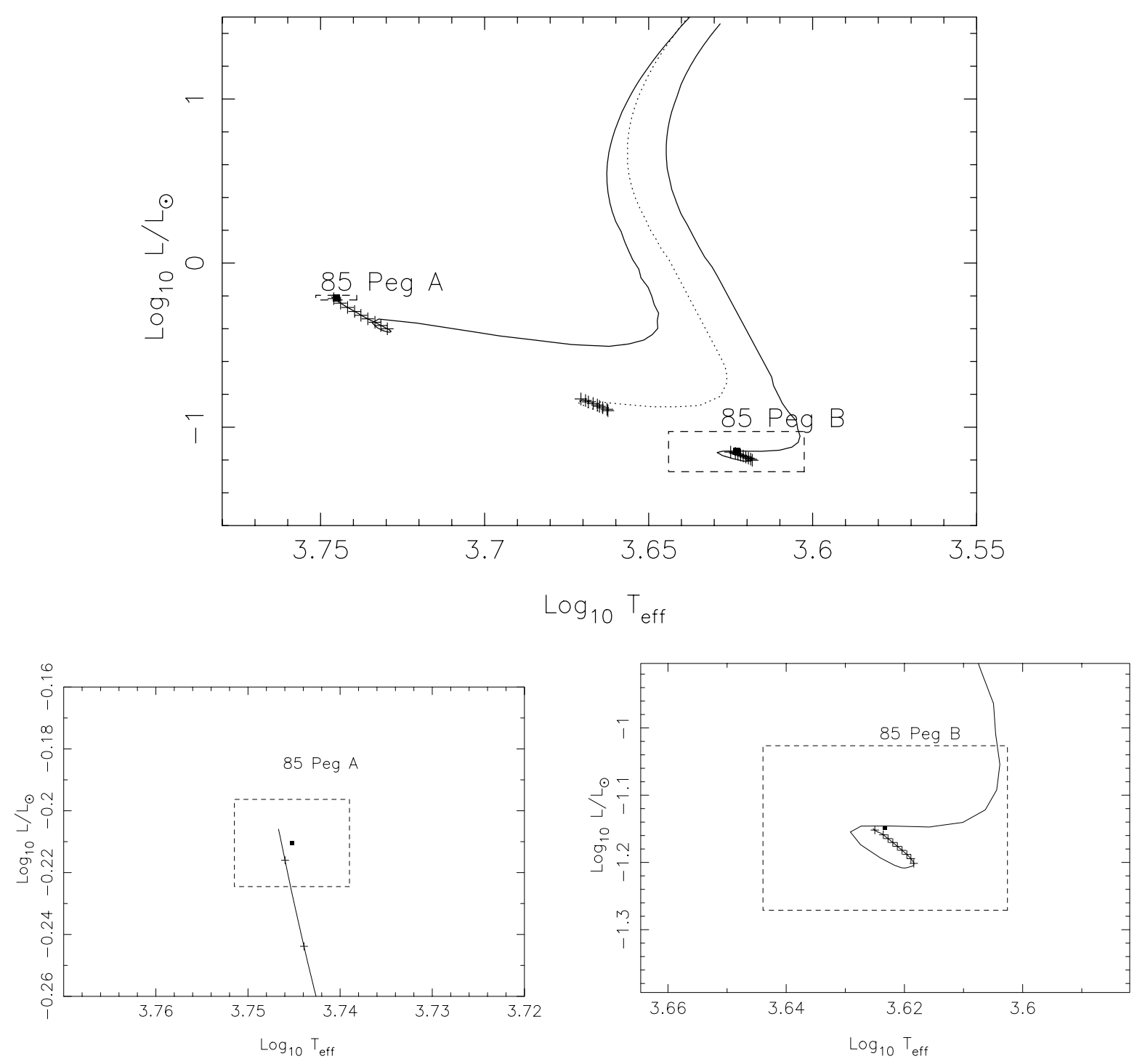

Fig. 1. Evolutionary tracks in the H-R diagram (see text). Dashed rectangles delimit the observed uncertainty domains. Top panel: full tracks from PMS, the dotted evolutionary track is for of a $0.66 M_{\odot}$ model computed with the calibration parameters of 85 Peg B (see text). The stellar evolution sequences are initialized on the pre-main sequence soon after the deuteron ignition. The "+" denote 1 Gyr time intervals along the evolutionary tracks. Bottom left and right panels: enlargements around the observed 85 Peg A, \& B loci.

track of a $0.66 M_{\odot}$ model computed with the same initial chemical composition and age. Our results give a mixinglength parameter for $85 \mathrm{Peg}$ A slightly lower than the solar one $\left(\Lambda_{\mathrm{A}}=1.8\right.$ against $\left.\Lambda_{\odot}=1.9\right)$. This could support the suggestion that the mixing-length parameter decreases with decreasing mass made by Lebreton et al. (2001) in the case of the Hyades cluster. This is however in disagreement with results from the 2D simulations where the mixing-length parameter appears to decrease with increasing effective temperature (Ludwig et al. 1999). According to our calibration 85 Peg is older than the Sun and is under-abundant in helium with respect to the Sun, which is consistent with what is expected for an old star in the Galaxy (Perrin et al. 1977). We point out that, due to element diffusion during evolution, our results show an observed metallicity at the surface of 85 Peg A and B considerably lower than the initial value, giving a 0.3 dex depletion in 9 Gyr.
Acknowledgements. We would like to express our thanks to the anonymous referee, for helpful comments. We would like to express our thanks to C. van't Veer, R. Cayrel, F. Thévenin and E. Lastennet for illuminating discussions and helpful advices. This research has made use of the Simbad data base, operated at CDS, Strasbourg, France. This work has been performed using the computing facilities provided by the OCA program "Simulations Interactives et Visualisation en Astronomie et Mécanique (SIVAM)". This work was partially supported by the "Convénio ICCTI-Embaixada de França" (B0-60) and by the project "PESO/P/PRO/15128/1999" from "Fundação para a Ciência e Tecnologia".

\section{References}

Alonso, A., Arribas, S., \& Martinez-Roger, C. 1996, A\&A, 291, 895

Axer, M., Fuhrmann, \& Gehren, T. 1994, A\&A, 291, 896

Baraffe, I., Chabrier, G., Allard, F., \& Hauschildt, P. H. 1998, A\&A, 337,403 
Bessell, M. S., Castelli, F., \& Plez, B. 1998, A\&A, 333, 231

Catchpole, R., Pagel, B., \& Powell, A. 1967, MNRAS, 136, 403

Duquennoy, A., \& Mayor, M. 1991, A\&A, 248, 485

Fernandes, J., Lebreton, Y., Baglin, A., \& Morel, P. 1998, A\&A, 338, 455

Flower, P. J. 1996, ApJ, 469, 355

Fulbright, J. P. 2000, AJ, 120, 1841

Fuhrmann, K. 1998, A\&A, 338, 161

Hall, R. J. Jr 1948, AJ, 54, 102

Heintz, W. D. 1993, AJ, 105, 1188

Henry, T. J., \& Mc Carthy, D. W. 1993, AJ, 106, 773

Kurucz, R. L. 1991, in Stellar Atmospheres: Beyond Classical Models, ed. L. Crivallery, I. Hibeny, \& D. G. Hammer, NATO ASI Series (Kluwer, Dordrecht)

Lastennet, E., Valls-Gabaud, D., Lejeune, Th., \& Oblak, E. 1999, A\&A, 349, 485

Lejeune, Th., Cusenier, F., \& Buser, R. 1998, A\&A, 130, 65

Lebreton, Y., Perrin, M.-N., Cayrel, R., Baglin, A., \& Fernandes, J. 1999, A\&A, 350, 587
Lebreton, Y., Fernandes, J., \& Lejeune, T. 2001, A\&A, 374, 540

Ludwig, H. G., Freytag, B., \& Steffen, M. 1999, A\&A, 346, 111

Martin, C., \& Mignard, F. 1998, A\&A, 330, 585

Morel, P. 1997, A\&AS, 124, 597

Morel, P., Provost, J., Lebreton, Y., Thévenin, F., \& Berthomieu, G. 2000, A\&A, 363, 675

Perrin, M.-N., Hejleson, P. M., Cayrel de Strobel, G., et al. 1977, A\&A, 54, 779

Slocum, F. 1915, ApJ, 41, 273

Smak, J. 1960, Acta Astron., 10, 153

Söderhjelm, S. 1999, A\&A, 341, 121

ten Brummelaar, T., Mason, B., McAlister, H., et al. 2000, AJ, 119, 2403

Thévenin, F., \& Idiart, T. 1999, ApJ, 521, 753

Thévenin, F. 2001, private communication van't Veer, C. 2000, private communication

Underhill, A. B. 1963, Pub. Dominion Astrophys. Obs., 12, 159

Wallerstein, G., \& Helfer, H. L. 1959, ApJ, 129, 720

Wyller, A. A. 1956, AJ, 61, 76 